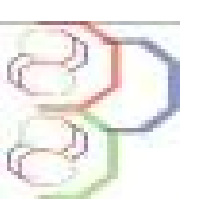

Journal of Applied Biosciences 92:8570 - 8577

ISSN 1997-5902

\title{
Étude de l'influence du facteur antécédent cultural palmiers et cocotiers sur l'évolution de la fusariose vasculaire chez six clones de palmiers à huile de Côte d'Ivoire
}

\author{
Sékou Diabaté1, Kouadio Laetitia Demby'1,4, Brou Kouassi Guy1,2, Yte Wongbe1, Konan Jean Noel3, \\ Konan Kouakou Eugene 3 , Abo Kouabenan 4 . \\ ${ }^{1}$ Labaoratoire de phyto/physiopathologie, Laboratoire Central de Biotechnologies, Centre National de Recherche \\ Agronomique (CNRA), 01 BP 1740 Abidjan 01, Côte d'Ivoire. \\ 2Laboratoire de Biologie et Amélioration des Productions Végétales, UFR-Science de la Nature, Université Nangui \\ Abrogoua (UNA), 02 BP 801 Abidjan 02. \\ ${ }^{3}$ Laboratoire de Cultures in vitro Lamé, Centre National de Recherche Agronomique (CNRA) ,13 BP 889 Abidjan 13. \\ ${ }^{4}$ Laboratoire de phytopathologie et de biologie végétale INPHB, BP 1313 Yamoussoukro. \\ Auteur correspondant, E-mail : sekou_diabate2002@yahoo.fr
}

Original submitted in on $8^{\text {th }}$ May 2015. Published online at www.m.elewa.org on $31^{\text {st }}$ August 2015

http://dx.doi.org/10.4314/jab.v92i1.1

\begin{abstract}
RESUME
Objectif : La fusariose vasculaire du palmier à huile, causée par le champignon Fusarium oxysporum f.sp. elaeidis, est une contrainte pathologique majeure pour la culture du palmier à huile en Afrique, notamment en Côte d'Ivoire. Dans la zone dite "savane de Dabou », en Côte d'Ivoire où la maladie sévit de manière endémique, les travaux de recherche de lutte intégrée à la fusariose, ont été réalisés dans le cadre de cette étude sur des parcelles à antécédent cultural cocotier ou palmier. Aussi, notre étude a-t-elle pour objectif général de confirmer les résultats de recherche obtenus pour l'amélioration du matériel végétal en vue de minimiser l'impact de la fusariose en Côte d'Ivoire. Spécifiquement, il s'est agi d'étudier l'évolution de la fusariose d'une part, et d'analyser cette évolution selon l'antécédent cultural d'autre part. Méthodologie et résultats : L'étude a porté sur l'observation des symptômes de fusariose cumulée et du taux de rémission de la fusariose des plants de palmier plantés de 1990 à 2013 sur antécédents cocotiers et palmers fusariés. Ainsi, pour la première fois, nos résultats révèlent que les palmiers replantés sur antécédents cocotiers manifestent plus rapidement la fusariose que ceux plantés sur antécédents palmiers. II est donc possible que les souches et les racines des cocotiers conservent le champignon pathogène de la première génération de palmiers fusariés constituant par conséquent une source d'inoculum lors des replantations.

Conclusion et application des résultats : Les sites à antécédents culturaux cocotier ne constituent pas un environnement agro écologique favorable pour la culture des plants de palmiers à huile sensibles à la fusariose. II est souhaitable d'utiliser des plants tolérants pour la mise en place de palmeraies industrielles. Mots clés : Palmier à huile, Cocotier, Fusarium oxysporum f.sp elaeidis, Clone, Antécédent cultural.
\end{abstract}




\begin{abstract}
Objective: The Fusarium wilt of oil palm, caused by the fungus Fusarium oxysporum $f$ elaeidis is a major pathological constraint to oil palm cultivation in Africa, particularly in Ivory Coast. In the area called "Dabou savannah" in Ivory Coast where the disease is endemic, IPM research to Fusarium wilt, was made study plots in cultural history coconut or palm tree. Also, this study general objective was to confirm the research findings for the improvement of plant material in order to minimize the impact of Fusarium in Ivory Coast. Specifically, it came to study the evolution of Fusarium wilt and analyze this development according to the cultural antecedent.

Methodology and results: The study focused on observation of symptoms of Fusarium and cumulative remission rate of Fusarium wilt on palm seedlings planted from 1990 to 2013 on cultural antecedent palmers and coconut infected by Fusarium oxysporum f.sp elaeidis. Thus, for the first time, our results reveal that planted on antecedent cultural coconut manifest Fusarium wilt faster than those planted on antecedent cultural palms. It is therefore possible that the stem and roots of coconut trees retain the pathogenic fungus of the first generation of fusarium palms constituting therefore a source of inoculum during replanting.

Conclusion and application of results: Sites of antecedent cultural coconut are not agro ecological environment favorable for the cultivation of oil palm seedlings susceptible to Fusarium. It is desirable to use tolerant clone for the establishment of industrial palm.
\end{abstract}

Keywords: Oil palm, coconut tree, Fusarium oxysporum f.sp elaeidis, Clone, cultural antecedent

\section{INTRODUCTION}

La filière palmier à huile (Elaeis guineensis) occupe une place de choix dans la société et l'économie Ivoirienne. Introduite en Côte d'Ivoire dans le cadre de la politique de diversification des produits de rente initiée depuis l'indépendance, cette filière a produit $400000 \mathrm{t}$ d'huile de palme (Fao stat, 2013) et dispose de 215000 hectares de palmeraie (Hubert, 2010). Toutefois, le palmier à huile fait l'objet de nombreuses attaques de ravageurs mais aussi de pathogènes. Au nombre des agents phytopathogènes figure Fusarium oxysporum f.sp. elaeidis, qui est l'agent causal de la fusariose vasculaire du palmier à huile. Dans des conditions écologiques favorables, des productions de plus de 6 tonnes d'huile par hectare/an peuvent être atteintes (Pamin et al., 1986 ; Escobar et al., 1996). Cependant, cette performance de production est jusque-là loin d'être généralisée compte tenu de la dispersion de l'agent pathogène dans le sol et de l'importance des superficies de cultures contaminée. Les méthodes conventionnelles de lutte (lutte chimique) pour le contrôle de cette trachéomycose, agent fongique tellurique spécifique du palmier à huile s'avèrent onéreuses et ne peuvent être économiquement envisageables. En effet plus de $50 \%$ des palmiers en production peuvent être atteints par la maladie si le matériel végétal est sensible à la maladie en situation de replantation (figure 1 et 2). En revanche, les méthodes de lutte préventives jusque-là adoptées se situent essentiellement au niveau du choix du matériel végétal après un test d'inoculation de l'agent pathogène au stade prépépinière (Allou et al., 2003; Diabaté et al., 2012). En outre, il est apparu très vite qu'à l'instar de certaines fusarioses, la sélection de matériel végétal tolérant à la maladie pouvait en limiter le développement (Dossa \& De Franqueville, 2010). Ainsi, une mission de recherche et de création de variétés résistantes à cette maladie a été assignée au Centre National de Recherche Agronomique (CNRA) qui poursuit les travaux de recherches entamés depuis 1961 par l'ex Institut de Recherche des Huiles et des Oléagineux (IRHO). Les travaux de recherche ont débuté par la sélection de croisements avant de s'étendre, à partir de 1980, au matériel clonal et, depuis les années 2000, à la sélection complémentaire aux approches classiques basées sur la synthèse de 
Diabaté et al. J. Appl. Biosci. Étude de l'influence du facteur antécédent cultural palmiers et cocotiers sur l'évolution de la fusariose vasculaire chez six clones de palmiers à huile de Côte d'Ivoire

composés phénoliques, dont les flavonoïdes, de tolérance. La présente étude, dont l'objectif est d'évaluer l'influence du facteur antécédent cultural sur l'évolution de la fusariose vasculaire chez six cultivars de palmiers à huile de Côte d'Ivoire, a été réalisée à grande échelle dans la zone dite "savane de Dabou », zone endémique à la fusariose sur la plantation Robert Michaux du CNRA.

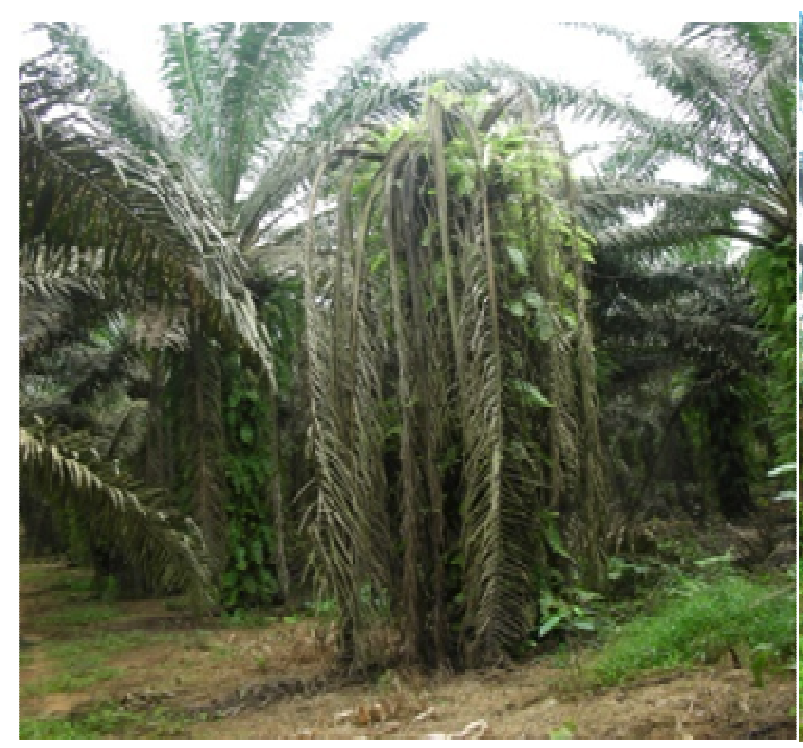

Figure 1 : Palmier atteint de fusariose aigue

\section{MATÉRIEL ET MÉTHODE}

Matériel végétal : Le matériel végétal est constitué d'hybrides (croisements) et de clones d'Elaeis guineensis. Ces cultivars ont été identifiés et nommés par le Centre National de Recherche Agronomique de

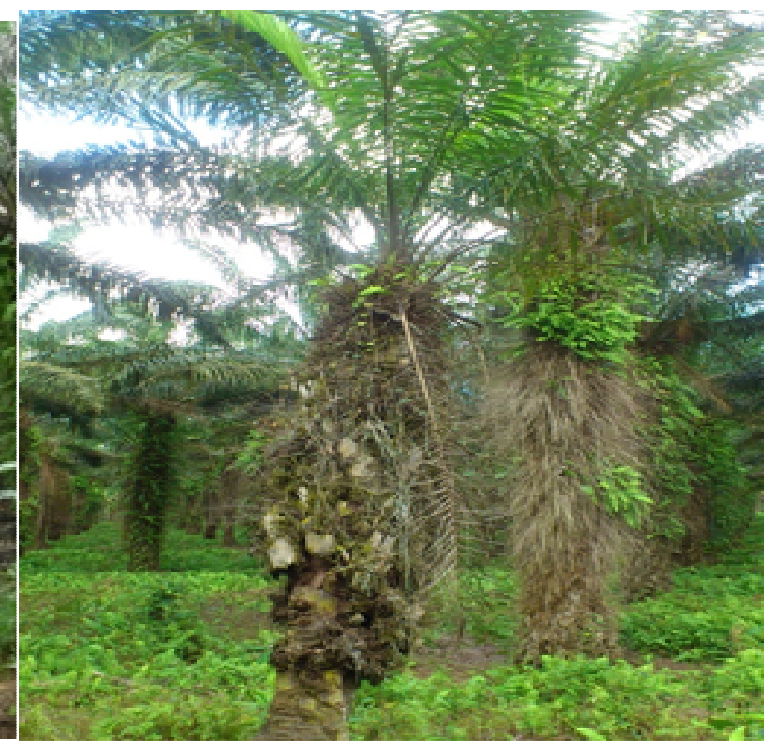

Figure 2 : Palmier atteint de fusariose chronique

Côte d'Ivoire (CNRA). Ils se composent de trois clones tolérants à la fusariose et de trois hybrides tolérants à la fusariose (Tableau 1 et 2).

Tableau 1 : Présentation des clones (CNRA, 2012)

\begin{tabular}{lll}
\hline Clones & Croisements (parents) & Indice fusariose \\
\hline LMC 022 & D 115D $\times$ L 2 T & 29 \\
LMC 063 & L 2 T $\times$ L269D & 79 \\
LMC 077 & D661D $\times$ L498P & 31 \\
\hline
\end{tabular}

Tableau 2 : Présentation des croisements (CNRA, 2012)

\begin{tabular}{llll}
\hline Numéro & Catégorie & Croisements & Indice fusariose \\
\hline S285 & C2501 & L9011D×L1574P & 65 \\
S295 & C2501 & L7803D×L2229P & 71 \\
S301 & C2501 & L9270D ×L2277P & 71 \\
\hline
\end{tabular}

Les clones LMC 022, LMC 063 et LMC 077ont des indices moyens de fusariose respectifs de 29,79 et 31 tandis que les hybrides S285; S295; S301 ont respectivement des indices moyens de 65,71 , et 71 .

Dispositif de mis en place des essais : Les clones et les hybrides ont été plantés et entretenus sur des parcelles à antécédents palmiers ou cocotiers avec huit répétitions par antécédent selon le dispositif en bloc de Fisher.

Méthode de collecte des données : Par année de culture, l'évolution de la fusariose sur le matériel végétal utilisé a été suivie depuis la mise en place des plants. Ainsi, les parcelles d'étude ont fait l'objet d'observations régulières de 1990 à 2013. La 
fréquence et les méthodes des relevés diffèrent selon l'âge de la plantation. Le relevé est un inventaire complet de tous les plants des différentes parcelles et des symptômes qu'ils présentent.

Fréquence des relevés de la fusariose : L'expression de la maladie peut être accentuée en replantation au niveau des jeunes cultures selon le comportement du matériel végétal. Ce qui suggère une fréquence de relevés plus importante qui s'établit comme suit :

- $\quad$ de 0 à 4 ans, une fois par trimestre ;
- $\quad$ de 5 à 10 ans, une fois chaque 4 mois ;
- $\quad$ de 11 à 25 ans, une fois chaque 6 mois.

Pourcentages de fusariose exprimée et cumulée : Les observations des symptômes permettent de suivre l'évolution de la fusariose de 1990 à 2013 sur les différentes parcelles et de déterminer régulièrement les taux de fusariose exprimée et cumulée.

- le pourcentage de fusariose exprimée, à la suite d'une observation donnée, comprend tous les palmiers qui ont exprimé des symptômes de fusarioses, y compris ceux qui sont morts de la maladie.

\section{RÉSULTATS ET DISCUSSION}

Évolution de la fusariose par antécédent cultural

Fusariose exprimée : Les pourcentages de fusariose exprimée selon l'antécédent cultural (Figure 1) ont présenté le taux maximum de $23 \%$ à l'année culturale de 1994. Ainsi, au niveau des parcelles à antécédent palmier, il est observé une évolution ascendante du taux de fusariose jusqu'en 1994 avant de subir une décroissance et de former un plateau à partir de 2004. De même, au niveau des parcelles à antécédent cocotier, il est constaté une évolution exponentielle du taux de fusariose jusqu'en 1994. Laquelle évolution est suivie d'une décroissance un an plus tard (1995) et de cela jusqu'en 2000 avant de décrire un plateau à partir de 2002, avec un taux d'environ $2 \%$. Ces résultats
- le pourcentage de fusariose cumulée comprend tous les palmiers qui ont exprimé, à un moment ou à un autre, les symptômes de la maladie qu'ils soient remis, fusariés ou morts.

Taux de rémission: C'est en considérant les pourcentages de fusariose exprimée et cumulée, et leur rapport, qu'il est possible d'établir un taux de rémission. Ce taux détermine la capacité du matériel végétal à surmonter l'agression parasitaire.

Analyses statistiques : La méthode d'analyse des résultats qui est associée le plus souvent au dispositif expérimental complètement aléatoire est l'analyse de variance à un critère de classification pour un seul facteur. Cependant, les données obtenues ne respectent pas les conditions d'application d'un test paramétrique; les analyses non-paramétriques sont donc les plus adaptées. Tous les tests statistiques ont été réalisés à l'aide du logiciel SPSS version 20. Les analyses sur le facteur antécédent cultural ont été réalisées par le test de Khi-deux au seuil de $5 \%$. Ce test permet de déterminer les liens de dépendance ou d'indépendance existant entre deux caractères qualitatifs.

corroborent le fait qu'en replantation, la fusariose est considérée comme une maladie du jeune âge (De Franqueville \& Diabaté, 1995; De Franqueville \& Renard, 1990 ; Nash et al, 1962). Cette expression de la maladie, au jeune âge, s'expliquerait par la forte pression d'inoculum à laquelle sont soumis les palmiers. En effet, les palmiers à huile de la première génération ayant fortement exprimé la fusariose, constituent une source importante d'inoculum dans la mesure où après la mort ou l'abattage du matériel végétal, les champignons se conservent sous forme de chlamydospores dans les souches (Allou, 1997 ; Allou et al., 2001). 


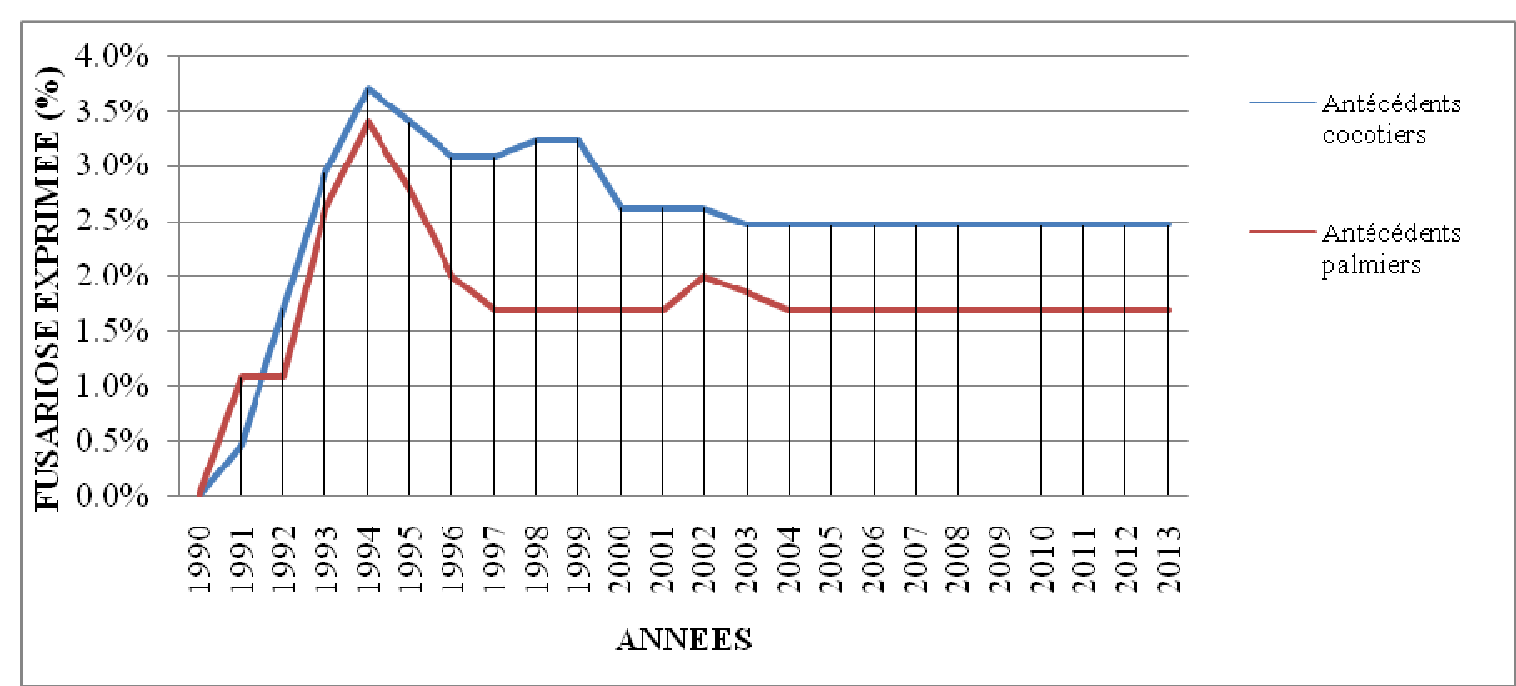

Figure 1: Évolution de la fusariose sur les blocs à antécédents palmiers et cocotiers

Le test du Khi-deux au seuil de $5 \%$ donne une signification asymptotique $(0,000)$ qui est inférieure à 0,05 . II y'a donc une différence hautement significative entre le nombre de palmiers atteint de fusariose de 1990 à 2013 selon l'antécédent cultural; les blocs ayant été replantés sur antécédent cocotier ont présenté un nombre de plants fusariés plus élevés que ceux à antécédent palmiers (Tableau 3). Selon Ataga et Van der Vossen (2007), cette sensibilité plus accrue des plants de palmiers sur les parcelles à antécédent palmier s'expliquerait par le fait que ces plants soient de même famille botanique contrairement aux plants de palmier mis en culture sur les parcelles à antécédent cocotier.

Tableau 3 : Relations entre les antécédents cocotiers et palmiers

\begin{tabular}{l|c|c|c}
\hline & Valeur & Ddl & Signification asymptotique (bilatérale) \\
\hline Khi-deux de Pearson & 97,303 & 48 & 0,000 \\
\hline Rapport de vraisemblance & 41,087 & 48 & 0,750 \\
\hline Association linéaire par linéaire & 11,443 & 1 & 0,001 \\
\hline Nombre d'observations valides & 23 & & \\
\hline
\end{tabular}

Fusariose cumulée par antécédent cultural de 1991 à 2013 : Le pourcentage de fusariose cumulée par antécédent cultural est représenté par la figure 2. Pour les parcelles à antécédent cocotier, le taux de fusariose cumulée subit une croissance exponentielle de 1990 à
2001 avant de se stabiliser à partir 2002. De même, chez celles à antécédent palmier, le taux de fusariose cumulée suit une croissance exponentielle de 1990 à 1998 avant de demeurer stable à partir de 1998. 


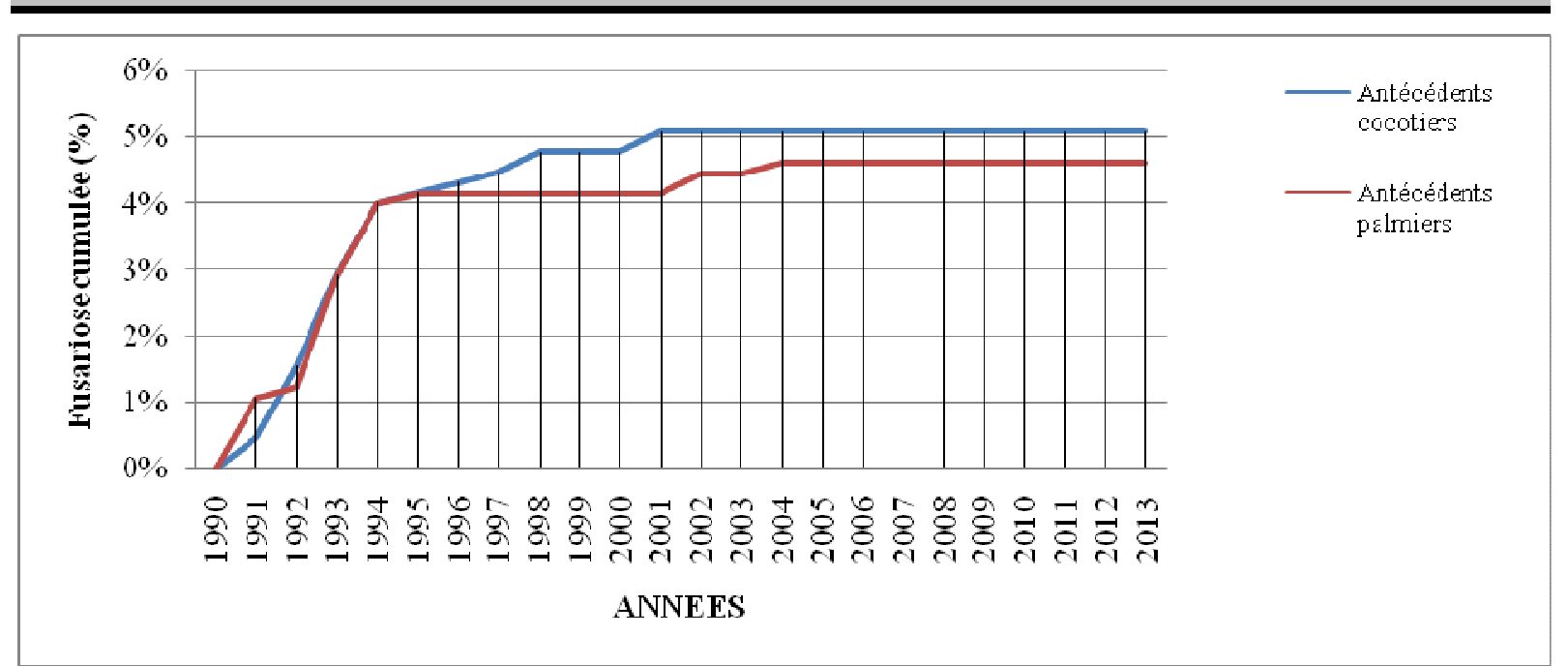

Figure 2: Évolution du pourcentage cumulée de la fusariose selon l'antécédent cultural

Toutefois, il y'a une différence hautement significative du pourcentage cumulé de palmiers atteint de fusariose de 1990 à 2013 selon l'antécédent cultural. Les blocs ayant été replantés sur antécédent cocotier ont présenté un pourcentage cumulé de plants fusariés plus élevés que ceux à antécédent palmiers (Tableau 4). Le test du Khi-deux au seuil de $5 \%$ donne une signification asymptotique $(0,000)$ qui est inférieure à 0,05 .

Tableau 4: Relations entre les cumuls des antécédents cocotiers et palmiers

\begin{tabular}{l|c|c|c}
\hline & Valeur & Ddl & Signification asymptotique (bilatérale) \\
\hline Khi-deux de Pearson & 110,198 & 48 & 0,000 \\
\hline Rapport de vraisemblance & 50,301 & 48 & 0,382 \\
\hline $\begin{array}{l}\text { Association linéaire par } \\
\text { linéaire }\end{array}$ & 21,152 & 1 & 0,000 \\
\hline $\begin{array}{l}\text { Nombre d'observations } \\
\text { valides }\end{array}$ & 23 & & \\
\hline
\end{tabular}

La succession cycles palmiers sensibles- cocotiers, n'a pas été bénéfique en replantation aussi bien pour les clones sensibles que pour les croisements tolérants replantés qui ont contractés la fusariose de manière significative au jeune âge. L'effet hautement dépressif de la fusariose sur l'ensemble du matériel végétal au stade juvénile s'expliquerait par le fait que les plantes, même les croisements tolérants, ont subi l'effet d'un inoculum primaire de Fusarium oxysporum f.sp elaeidis très abondant. Certains auteurs ont montré, en effet, que la population de Fusarium oxysporum dans la rhizosphère de différentes plantes ne dépendait pas uniquement de la plante cultivée, mais aussi du précédent cultural (Besri, 1975; Alabouvette et al, 1982). D'autres ont même montré les effets positifs de la rotation culturale sur la réceptivité du sol au champignon par la modification notable de la rhizosphère (Alabouvette, 1982; Allou et al., 2001).
Cependant, la rotation culturale impliquerait que les cycles de culture utilisés soient de famille botanique différente pour que cela soit profitable à la culture. Ainsi, Ataga et Van der Vossen (2007) ont révélé, dans le cas de la pourriture de la base du tronc des palmiers (causée par Ganoderma sp.), des pertes plus importantes lorsqu'on replantait des terrains qui ont eu comme précédent cultural le cocotier ou le palmier à huile. Ceci pourrait donc expliquer les pertes plus élevées dues à la fusariose en replantation de plants palmiers sur antécédent cocotier constatées dans la présente étude.

Taux de rémission par année selon l'antécédent cultural : Le taux de rémission de la fusariose par année selon l'antécédent cultural (Figure 3) a montré une croissance à évolution exponentielle jusqu'en 1998 pour se stabiliser à partir de l'année suivante (1999). 


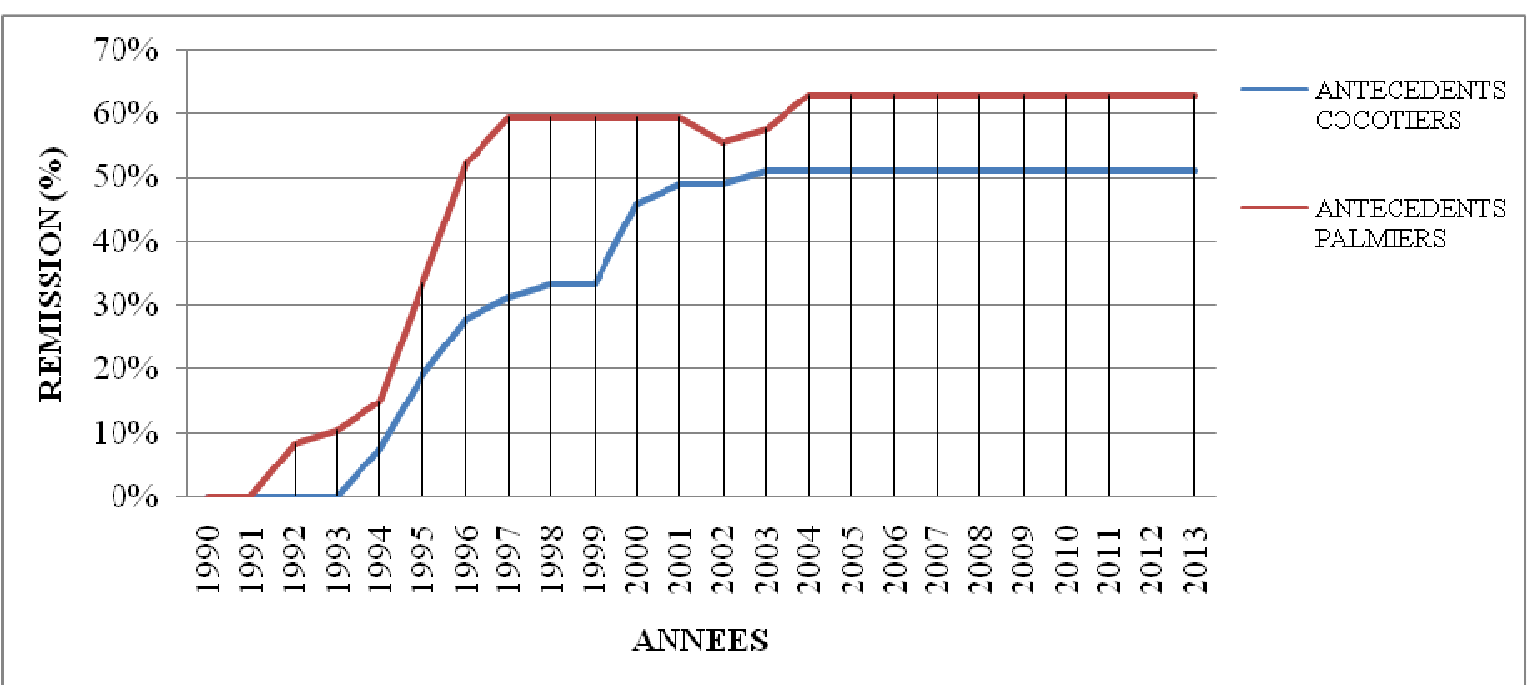

Figure 3: Évolution du taux de rémission par année selon l'antécédent cultural

Le test du Khi-deux au seuil de $5 \%$ donne une signification asymptotique $(0,000)$ et est inférieure à 0,05 . II y'a donc une différence hautement significative entre le pourcentage de plants remis de la fusariose de 1990 à 2013 selon l'antécédent cultural. Les blocs ayant été replantés sur antécédents palmiers, ont un taux de rémission supérieur à ceux des blocs replantés antécédents cocotiers (Tableau 5). L'obtention du taux positif de rémission à la fusariose serait lié aux croisements tolérants qui ayant manifesté la fusariose pendant les quatre premières années de plantation, se remettraient par la suite de la maladie (Diabaté et al., 2010).

Tableau 5 : Relations entre les taux de rémission des antécédents cocotiers et palmiers

\begin{tabular}{|l|c|c|c|}
\hline & Valeur & ddl & Signification asymptotique (bilatérale) \\
\hline Khi-deux de Pearson & 138,000 & 72 & 0,000 \\
\hline Rapport de vraisemblance & 67,205 & 72 & 0,638 \\
\hline Association linéaire par linéaire & 19,910 & 1 & 0,000 \\
\hline Nombre d'observations valides & 23 & & \\
\hline
\end{tabular}

\section{CONCLUSION}

II ressort de la présente étude que la succession de cycles palmiers - cocotiers, constitue un environnement agroécologique défavorable à la replantation de palmiers à huile en zones fusariées. Le Fusarium oxysporum f.sp elaeidis se conserverait dans les tissus résiduels de cocotier et de palmiers constituant ainsi un inoculum primaire en cas de replantations de ces sites.

\section{RÉFÉRENCES BIBLIOGRAPHIQUES}

Alabouvette C., Couteaudier y. et Louvet J., 1982: Comparaison de la réceptivité des sols à la fusariose du palmier à huile. Agronomie, 2(1) :1-6.

Allou K., 1997. La fusariose vasculaire du palmier à huile due à Fusarium oxysporum f. spelaedis, en zone de savane: cas de la plantation expérimentale Robert MICHAUX de Dabou.
Les clones et croisements testés ont manifesté la fusariose pendant les quatre première années de plantation avant de se remettre irréversiblement de la maladie. Toutefois, les résultats de ce travail ont montré que les replantations en zones fusariées doivent absolument être réalisées avec des croisements ou des clones tolérants à la fusariose.

Thèse de doctorat : université de Cocody. 155 p.

Allou K., Ahoussou N., Ake S., Diabate S., et De Franqueville H., 2003; Comportement De Clones De Palmier A Huile Au Champ En Zones De Haute Densité De Fusarium oxysporum f. sp. elaeidis en Côte d'lvoire. Agronomie Africaine 15(1) : 29-38 
Allou K., Ake S., Ahoussou N., Ballo K., et Diabate S., 2001, effet de la jachère sur l'expression de la fusariose vasculaire du palmier à huile (Elaeis guineensis Jacq.), Agronomie Africaine 13(1): 21-33.

Ataga C. D. et Van Der Vossen H., 2007, Elaeis guineensis, basé sur procea 1 : vegetable oils and fats, Oléagineux. P84.

De Franqueville et Renard., 1990. Bilan de l'amélioration du niveau de tolérance du palmier à huile à la fusariose Évolution de la maladie sur la plantation de Robert Michaux, oléagineux, octobre 1990, 399-401p.

De Franqueville H.et Diabaté S., 1995, La fusariose du palmier à huile en Afrique de l'Ouest, Plantations, recherche et développement, 510p.

Diabaté S., Ake S., Kouame K.R., Coulibaly O.A., et N'guessan W.P., 2010, phenolic diversity in defence reaction of the oil palm against vascular wilt disease, Agriculture and biology journal of north America 1(3): 407-415p.

Diabaté S, Gbongué L-R, Dick E, Bomisso E L and Franqueville (de) H : 2012. Étude Comparée de L'action de Souches Non Pathogènes de Fusarium oxysporum dans l'induction des réactions immunitaires du palmier à huile contre la fusariose. European Journal of Scientific Research 3(2) : 193-201.

Escobar R., Sterling F., and Peralta F: 1996. Oil Palm planting materials by ASD de Costa Rica. In: Étude de la diversité génétique du genre Elaeis ( $E$. oleifera (Kunth) Cortés et $E$. guineensis Jacq.) par marqueurs moléculaires (RFLP et AFLP), Thèse de Doctorat de l'école nationale supérieure Agronomique de Montpellier, $137 \mathrm{p}$.

FAO : 2013. Bilans alimentaire: production mondiale de I'huile de palme. Division de la statistique, FAOSTAT, Série informatique, FAO, Rome, Italie.

Hubert $0: 2010$. Contribution de la production d'huile de palme au développement durable Oléagineux, Corps gras, Lipides, 17(6) : 362367.

Nash S.M. et Snyder W.C., 1962.Quantitative estimations by plate counts of propagule of the bean root rot Fusarium in field soil, Phytopathology 52: 567-572.

Pamin K., Hutomo J., Syamsuddin E. and Purba AR : 1996. Potential productivity of IOPRI planting materials. In : Étude de la diversité génétique du genre Elaeis (E. oleifera (Kunth) Cortés et E. guineensis Jacq.) par marqueurs moléculaires (RFLP et AFLP), Thèse de Doctorat de l'école nationale supérieure Agronomique de Montpellier, 137 